\title{
Fast Algorithms for Signal Reconstruction without Phase
}

\author{
Radu Balan ${ }^{a}$, Bernhard G. Bodmann ${ }^{b}$, Peter G. Casazza ${ }^{c}$, Dan Edidin ${ }^{d}$ \\ ${ }^{a}$ Mathematics Department and CSCAMM, University of Maryland, \\ College Park, MD 20742, USA \\ ${ }^{b}$ Mathematics Department, University of Houston, \\ Houston, TX 77204, USA \\ ${ }^{c, d}$ Mathematics Department, University of Missouri, \\ Columbia, MO 65211, USA;
}

\begin{abstract}
We derive fast algorithms for doing signal reconstruction without phase. This type of problem is important in signal processing, especially speech recognition technology, and has relevance for state tomography in quantum theory. We show that a generic frame gives reconstruction from the absolute value of the frame coefficients in polynomial time. An improved efficiency of reconstruction is obtained with a family of sparse frames or frames associated with complex projective 2-designs.
\end{abstract}

Keywords: Frames, mutually unbiased bases, equiangular frames, projective 2-designs, discrete chirps

\section{INTRODUCTION}

For years engineers believed that if a signal was represented with a sufficient degree of redundancy, then its reconstruction should be possible without using the recorded phase information. Typical examples are the reconstruction of an unknown square-integrable function from the magnitude of its windowed Fourier Transform, also known as the short-time Fourier transform, or of an undecimated wavelet transform in audio and image processing. One motivation for studying this problem arose from speech recognition technology where information about the phase of a signal is lost in the course of processing using cepstral analysis (see $[3,6,29,30,32,35]$ ).

The problem of reconstruction without phase appears also in the context of quantum theory, where a quantum state is determined by measuring correlations with other states [28]. More precisely, a pure quantum state is given by a ray in a complex Hilbert space, and is usually represented by normalized vectors in this ray. Quantum measurements only give access to the magnitudes of the inner product between these vectors with other state vectors. Therefore, reconstructing a pure quantum state from measurements is the same as finding a vector, up to a unimodular constant, from the magnitudes of linear transform coeffients. This is also referred to as quantum state tomography [31].

Moreover, there is a closely connected problem in optics with applications to X-ray, crystallography, electron microscopy, and coherence theory (see $[5,18,19,27]$ ). This problem is to reconstruct a discrete signal from the modulus of its Fourier transform under constraints in both the original and the Fourier domain. For finite signals the approach uses the Fourier transform with redundancy 2. All signals with the same modulus of the Fourier transform satisfy a polynomial factorization equation. In dimension one this factorization has an exponential number of possible solutions. In higher dimensions the factorization is shown to have generically a unique solution (see $[22])$.

Recently, the problem of reconstruction without phase was solved for a large class of frames in finitedimensional Hilbert spaces [3]. It is easily seen that, in the case of a real Hilbert space, this problem is equivalent

Further author information: (Send correspondence to P. Casazza)

R.B.: E-mail: rvbalan@math.umd.edu

B.G.B: E-mail: bgb@math.uh.edu

P.G.C.: E-mail: pete@math.missouri.edu, Telephone: 15738828285

D.E.: E-mail: edidin@math.missouri.edu, Telephone: 15738827475 
to a combinatorial problem. In [4] it is shown that signal reconstruction from the absolute value of the frame coefficients is equivalent to the solution of a sparse signal optimization problem. In particular, this task can be phrased as minimizing an $\ell^{p}$ (quasi)norm over a linear constraint.

Unfortunately, the numerical implementation of such results has been hampered by a lack of good algorithms for doing signal reconstruction from the absolute value of the frame coefficients. The purpose of this paper is to introduce several classes of fast algorithms for doing signal reconstruction without phase.

Our goal is to derive simple algorithms for doing signal reconstruction without phase. We will use a variety of tools for this including generic frames, projective 2-designs, which include equiangular tight frames and mutually unbiased bases, in particular discrete chirps. Each of the list of algorithms has its own advantage for the problem at hand. In [3] it is shown that a generic frame with $4 N-2$-elements in an $N$-dimensional complex Hilbert space will yield signal reconstruction from the absolute value of the frame coefficients. In the real case the frames must have size $2 N-1$. We will see here that a generic frame will give signal reconstruction without phase in polynomial time. In another paper [2] we construct frames which yield linear reconstruction algorithms. The organization of the paper is as follows. In section 2 we introduce the necessary results from frame theory including the classes of frames we will be using. Section 3 gives the results concerning generic frames. Section 4 gives fast algorithms for sparse frames. Section 5 contains a review of algorithms from [2] using projective 2-designs. Section 6 contains a review of algorithms from [2] using mutually unbiased bases. Section 7 is a discussion of linear reconstruction algorithms. Section 8 contains concluding remarks.

\section{FRAME THEORY}

We now introduce the fundamental notions from frame theory used in the paper. The main property of frames which makes them so useful in applied problems is their redundancy. That is, each vector in the space has infinitely many representations with respect to the frame but it also has one natural representation given by the frame coefficients. The role played by redundancy varies with specific applications. In general, a carefully chosen frame can provide resilience to quantization, resilience to additive noise, numerical stability for reconstruction and an ability to capture important signal characteristics. A family of vectors $\left\{f_{i}\right\}_{i \in I}$ is a frame for a Hilbert space $\mathbb{H}$ (with lower and upper frame bounds $0<A \leq B<\infty$ respectively) if for all $x \in \mathbb{H}$ :

$$
A\|x\|^{2} \leq \sum_{i \in I}\left|\left\langle x, f_{i}\right\rangle\right|^{2} \leq B\|x\|^{2} .
$$

The numbers $\left\{\left\langle x, f_{i}\right\rangle\right\}_{i \in I}$ are called the frame coefficients. If $A=B$ this is a $A$-tight frame and if $A=B=1$, it is called a Parseval frame. If all the frame elements have the same norm we call this an equal-norm frame. Given a frame $\left\{f_{i}\right\}_{i \in I}$, the analysis operator is $T: \mathbb{H} \rightarrow \ell^{2}(I)$ given by: $T(x)=\left\{\left\langle x, f_{i}\right\rangle\right\}_{i \in I}$. The range of $T$ is called the range of coefficients. The synthesis operator is the adjoint of $T$, and satisfies

$$
T^{*}(c)=\sum_{i \in I} c_{i} f_{i}
$$

The frame operator is the positive, self-adjoint invertible operator $S=T^{*} T: \mathbb{H} \rightarrow \mathbb{H}$.

We refer the reader to [16] for an introduction to frame theory.

We will need several special classes of frames which we now introduce.

Definition 2.1. A family of vectors $\mathcal{F}=\left\{f_{j}\right\}_{j=1}^{M}$ is said to form a 2-uniform A-tight frame if it is equal-norm and if there is $c>0$ such that for all pairs of frame vectors $f_{j}$ and $f_{k}, j \neq k$, we have $\left|\left\langle f_{j}, f_{k}\right\rangle\right|=c$.

It is well known that there is an upper bound for the number of vectors in a tight 2-uniform frame $\mathcal{F}=\left\{f_{j}\right\}_{j=1}^{M}$ on an $N$-dimensional Hilbert space $\mathbb{H}$. In the case of a real $\mathbb{H}, M \leq N(N+1) / 2$, and in the complex case, $M \leq N^{2}$. (See [23,2]). The construction of the maximal number of vectors for a tight 2-uniform frame is a very deep and difficult open problem in frame theory. 
Definition 2.2. Let $\mathbb{H}$ be a real or complex Hilbert space. A family of vectors $\left\{e_{k}^{(j)}\right\}$ in $\mathbb{H}$ indexed by $k \in \mathbb{K}=$ $\{1,2, \ldots N\}$ and $j \in \mathbb{J}=\{1,2, \ldots M\}$ is said to form $\mathrm{M}$ mutually unbiased bases if for all $j, j^{\prime} \in \mathbb{J}$ and $k, k^{\prime} \in \mathbb{K}$ the magnitude of the inner product between $e_{k}^{(j)}$ and $e_{k^{\prime}}^{\left(j^{\prime}\right)}$ is given by

$$
\left|\left\langle e_{k}^{(j)}, e_{k^{\prime}}^{\left(j^{\prime}\right)}\right\rangle\right|=\delta_{k, k^{\prime}} \delta_{j, j^{\prime}}+\frac{1}{\sqrt{N}}\left(1-\delta_{j, j^{\prime}}\right),
$$

with Kronecker's symbol $\delta_{j, j}=1$ and $\delta_{j, j^{\prime}}=0$ if $j \neq j^{\prime}$.

There are at most $M=N+1$ mutually unbiased bases $\left\{e_{k}^{j}: 1 \leq j \leq M, 1 \leq k \leq N\right\}$ in an $N$-dimensional complex Hilbert space $\mathbb{H}[$ See 17, 2]. The construction of the maximal number of mutually unbiased bases for Hilbert spaces of arbitrary dimensions is also a deep and difficult open problem in frame theory. However, it is possible for $N$ a prime power in the complex case and a power of 4 in the real case. (See $[9,10]$ ).

Examples of mutually unbiased bases are given by the discrete chirps.

Proposition 2.3. Let $N$ be a prime number, $\omega$ a primitive $N$-th root of unity, and denote the canonical basis of $\mathbb{C}^{N}$ by $\left\{e_{k}\right\}_{k=1}^{N}$. Then we identify $e_{k}^{(1)} \equiv e_{k}$ and for $j \in\{2,3, \ldots N+1\}$ let

$$
e_{k}^{(j)}=\frac{1}{\sqrt{N}} \sum_{l=1}^{N} \omega^{-(j-1) l^{2}+k l} e_{l} .
$$

This defines a family of $N+1$ mutually unbiased bases that has been called the discrete chirps [11, 24].

\section{GENERIC FRAMES}

In this section we will show that a generic frame will give signal reconstruction without phase and will achieve this in polynomial time. First we will need to define generic.

We consider the non-linear mapping taking a vector to the absolute value of its frame coefficients:

$$
\mathbb{M}: H \rightarrow \ell^{2}(I), \mathbb{M}(x)=\left\{\left|\left\langle x, f_{i}\right\rangle\right|\right\}_{i \in I}
$$

If it is necessary to associate this to its frame we will write $\mathbb{M}^{\mathcal{F}}$. Let $H_{r}=H / \sim$ be the quotient space obtained by identifying two vectors if they differ by a constant phase factor. That is, $x \sim y$ means there is a scalar $|c|=1$ so that $y=c x$. For real Hilbert spaces, $c= \pm 1$ while for complex Hilbert spaces $c=e^{i \theta}$. In quantum mechanics, these projective rays define quantum states [33]. The nonlinear mapping $\mathbb{M}$ extends to $H_{r}$ as

$$
\mathbb{M}: H_{r} \rightarrow \ell^{2}(I), \mathbb{M}(\hat{x})=\left\{\left|\left\langle x, f_{i}\right\rangle\right|\right\}_{i \in I}, x \in \hat{x}
$$

First consider the case $\mathbb{H}=\mathbb{R}^{N}$ and let $l^{2}(I)$ be $\mathbb{R}^{M}$. The set $\operatorname{Gr}(N, M ; \mathbb{R})$ of $N$-dimensional linear subspaces of $\mathbb{R}^{M}$ has the structure of an $N(M-N)$-dimensional manifold called the Grassmann manifold [36, p. 129]. The frame bundle $F(N, M ; \mathbb{R})$ is the $G L(N, \mathbb{R})$-bundle over $G r(N, M)$ defined as follows: The fiber of $F(N, M ; \mathbb{R})$ over a point of $G L(N, \mathbb{R})$ corresponding to an $N$-dimensional linear subspace $W \subset \mathbb{R}^{M}$ is the set of all possible bases for $W$.

For a frame $\mathcal{F}=\left\{f_{1}, \ldots, f_{M}\right\}$ of $\mathbb{R}^{N}$ the analysis operator $T$ satisfies

$$
T: \mathbb{R}^{N} \rightarrow \mathbb{R}^{M}, T(x)=\sum_{k=1}^{M}\left\langle x, f_{k}\right\rangle e_{k},
$$

where $\left\{e_{1}, \ldots, e_{M}\right\}$ is the canonical basis of $\mathbb{R}^{M}$. We let $W$ denote the range of the analysis map $T\left(\mathbb{R}^{N}\right)$. It is an $N$-dimensional linear subspace of $\mathbb{R}^{M}$ and thus corresponds to a point of the Grassman manifold $G r(N, M)$. Two frames $\left\{f_{i}\right\}_{i \in I}$ and $\left\{g_{i}\right\}_{i \in I}$ are equivalent if there is an invertible operator $T$ on $H$ with $T\left(f_{i}\right)=g_{i}$, for all $i \in I$. It is known that two frames are equivalent if and only if their associated analysis operators have the same range (see $[1,21]$ ). We deduce that $M$-element frames on $\mathbb{R}^{N}$ are parametrized by the fiber bundle $F(N, M ; \mathbb{R})$. 
In [3] it is shown that two equivalent frames $\mathcal{F}$ and $\mathcal{G}$ have the property that $\mathbb{M}^{\mathcal{F}}$ is injective if and only if $\mathbb{M}^{\mathcal{G}}$ is injective. It follows that for two frames corresponding to two points in the same fiber of $F(N, M ; \mathbb{R})$, the injectivity of their associated nonlinear maps would jointly hold true or fail.

The complex case $\mathbb{H}=\mathbb{C}^{N}$ behaves basically the same way. The Hilbert space is $\mathbb{C}^{N}$. For an $M$-element frame $\mathcal{F}=\left\{f_{1}, \ldots, f_{M}\right\}$ of $\mathbb{C}^{N}$ the analysis operator is defined by (3), where the scalar product is $\langle x, y\rangle=\sum_{k=1}^{N} x_{k} \overline{y_{k}}$. The range of coefficients, i.e. the range of the analysis operator, is a complex $N$-dimensional subspace of $\mathbb{C}^{M}$ that we denote again by $W$. Thus a frame determines a point of the complex Grassmanian $G r(N, M)^{\mathbb{C}}$ parametrizing $N$-dimensional complex subspaces of $\mathbb{C}^{N}$. As in the real case, the set of $M$-frames of $\mathbb{C}^{N}$ is parametrized by points of the fiber bundle $F(N, M ; \mathbb{C})$, the $G L(N, \mathbb{C})$-bundle over $G r(N, M)^{\mathbb{C}}$.

The nonlinear map we are using is given by

$$
\mathbb{M}^{\mathcal{F}}: \mathbb{C}^{N} / \mathbb{T}^{1} \rightarrow \mathbb{C}^{M} \quad, \quad \mathbb{M}^{\mathcal{F}}(\hat{x})=\sum_{k=1}^{M}\left|\left\langle x, f_{k}\right\rangle\right| e_{k} \quad, \quad x \in \hat{x}
$$

where two vectors $x, y \in \hat{x}$ if there is a scalar $c \in \mathbb{C}$ with $|c|=1$ so that $y=c x$.

The main result of [3] is then:

THEOREM 3.1. The following hold:

(Real frames) If $M \geq 2 N-1$, then for a generic frame $\mathcal{F}$, the nonlinear map $\mathbb{M}$ is injective.

(Complex frames) If $M \geq 4 N-2$ then for a generic frame $\mathcal{F}$, the nonlinear map $\mathbb{M}$ is injective.

REMARK 3.2. In both the real and complex case of Theorem 3.1 the term "generic" means following: If we view the Grassmannian $\operatorname{Gr}(N, M)$ as a real algebraic variety then there is a Zariski open set $U \subset G r(N, M)$ such that the conclusion holds for all frames whose associated linear subspace corresponds to a point of $U$.

In the real case, if $M \leq 2 N-2$, then no frame $\left\{f_{i}\right\}_{i=1}^{M}$ can satisfy the theorem (See [3]). We do not know the exact minimal bound for the complex case. Also, in the real case there is a simple direct method for checking if $\mathbb{M}$ is injective for a given frame [3].

TheOREM 3.3. Let $\left(f_{i}\right)_{i=1}^{M}$ be a frame for $\mathbb{R}^{N}$. The following are equivalent:

(1) The map $\mathbb{M}$ is injective.

(2) For every subset $\phi \subset\{1,2, \ldots, M\}$, either $\left\{f_{i}\right\}_{i \in \phi}$ spans $\mathbb{R}^{N}$ or $\left\{f_{i}\right\}_{i \in \phi^{c}}$ spans $\mathbb{R}^{N}$.

Corollary 3.4. If $\mathcal{F}$ is a $M$-element frame for $\mathbb{R}^{N}$ with $M \geq 2 N-1$ having the property that every $N$-element subset of the frame is linearly independent, then $\mathbb{M}$ is injective.

Now we will show that a generic frame will give signal reconstruction without phase in a polynomial number of steps. Let $\mathbb{H}$ be a fixed $N$-dimensional vector space which is assumed to be either real or complex and let $\left\{e_{1}, \ldots e_{N}\right\}$ be a chosen orthonormal basis for $\mathbb{H}$. We will henceforth refer to this as the "standard basis" for $\mathbb{H}$.

ThEOREM 3.5. (a) If $\mathbb{H}$ is real, $M \geq \frac{N(N+1)}{2}$ and $\mathcal{F}=\left\{f_{1}, \ldots, \ldots f_{M}\right\}$ is a generic frame and $x \in \mathbb{H}$ is a vector then $x$ can be reconstructed (up to sign) from the set $\left\{\left|\left\langle x, f_{i}\right\rangle\right|\right\}_{i=1}^{M}$ of absolute values of its frame coefficients in a polynomial number $\left(O\left(N^{6}\right)\right)$ of steps.

(b) If $\mathbb{H}$ is complex and $M \geq N^{2}$ and $\mathcal{F}=\left\{f_{1}, \ldots, f_{M}\right\}$ is a generic frame then a vector $x \in \mathbb{H}$ can be reconstructed (up to multiplication by a root of unity) from the set $\left\{\left|\left\langle v, f_{i}\right\rangle\right|\right\}_{i=1}^{M}$ of moduli of the frame coefficients in a polynomial number $\left(\left(O\left(N^{6}\right)\right)\right.$ of steps.

In both the real and complex cases the term "generic" is used in the sense of Remark 3.2

Proof. Since $\mathcal{F}$ is a frame, we know that some $N$-element subset of $\mathcal{F}$ spans $\mathbb{H}$. To simplify our notation we will reorder the vectors so that we may assume that $\left\{f_{1}, \ldots, f_{N}\right\}$ span $\mathbb{H}$. By inverting the $N \times N$ matrix whose rows are $f_{1}, \ldots, f_{N}$ (which we can in $O\left(N^{3}\right)$ steps) we may expand our other frame vectors as $f_{k}=\sum_{i=1}^{N} u_{k, i} f_{i}$.

Let $x$ be a vector and let $\left\{a_{1}, \ldots, a_{M}\right\}$ be the frame coefficients of $x$ relative to $\mathcal{F}$; i.e. $a_{i}=\left\langle x, f_{i}\right\rangle$ for all $i=1,2, \ldots, M$. Then for $k>N, a_{k}=\sum_{i=1}^{N} u_{k, i} a_{i}$. We wish to determine the $a_{i}$ 's (up to a global multiplication 
by a root of unity in the ground field). Choose $i \leq N$ such that $a_{i} \neq 0$ (such $i$ exists because $\left\{f_{1}, \ldots, f_{N}\right\}$ span $\mathbb{H})$ and let $b_{j}=a_{j} / a_{i}$ for $j=1,2, \ldots, M$. Determining the $a_{j}$ 's up to to global multiplication by a root of unity is obviously equivalent to computing the $b_{j}$ 's exactly.

Our method is to compute $\left\{b_{1}, \ldots b_{N}\right\}$ (and thus the other $b_{j}$ 's) using Guassing elimination. To simplify the notation we reorder the vectors and assume that $b_{1}=1$. We now distinguish between the real and complex case.

Real Case: For each $k \geq N$ we know $b_{k}^{2}$. Expanding in terms of the $b_{i}$ 's for $i \leq N$ we obtain the equation

$$
\begin{aligned}
b_{k}^{2} & =\left(\sum_{i=1}^{N} u_{k, i} b_{i}\right)^{2} \\
& =\sum_{i=1}^{N} u_{k, i}^{2} b_{i}^{2}+\sum_{1<i<j \leq N} 2 u_{k, i} u_{k, j} b_{i} b_{j}+\sum_{i=1}^{N} u_{k, i} u_{k, 1} b_{i},
\end{aligned}
$$

which we may view as a linear equation in the $(N-1)+\frac{(N-1)(N-2)}{2}$ unknowns $\left\{b_{i}\right\}_{i=2, \ldots, N}$ and $\left\{b_{i} b_{j}\right\}_{1<i<j \leq N}$. By (Theorem 2.2, [3]) for a generic frame the linear system of equations given by $b_{N+1}, \ldots b_{N(N+1) / 2}$ has a unique solution (since the $b_{k}$ 's are uniquely determined by their absolute values). This solution can be obtained by Gaussian elemination requring $O\left(\left(N^{2}\right)^{3}\right)$ steps. Once the $b_{i}$ 's are determined we can reconstruct $x$ (up to multiplication by a root of unity) by expressing the basis $f_{1}, \ldots f_{N}$ in terms of the given orthonormal basis. This requires $O\left(N^{3}\right)$ steps.

Complex Case: In the complex case we expand

$$
\begin{aligned}
\left|b_{k}\right|^{2} & =\left.\left(\sum_{i=1}^{N} \mid u_{k, i} b_{i}\right)\right|^{2} \\
& =\sum_{i=1}^{N}\left|u_{k, i}\right|^{2}\left|b_{i}\right|^{2}+\sum_{1<i<j \leq N} u_{k, i} \overline{u_{k, j}} b_{i} \overline{b_{j}} \\
& +\sum_{1<i<j \leq N} \overline{u_{k, i}} u_{k, j} \overline{b_{i}} b_{j}+\sum_{i=1}^{N} u_{k, i} u_{k, 1} b_{i}+\sum_{i=1}^{N} \overline{u_{k, i} u_{k, 1}} \overline{b_{i}}
\end{aligned}
$$

which we may view as a linear equation in the $2(N-2)+(N-1)(N-2)$ unknowns given by $\left\{b_{i}, \overline{b_{i}}\right\}_{i=2, \ldots, N}$ and $\left\{b_{i} \overline{b_{j}}, \overline{b_{i}} b_{j}\right\}_{1<i<j \leq N}$. Once again invoking (Theorem 2.2, [3]) for a generic frame this system has a unique solution which can be obtained in $O\left(\left(N^{2}\right)^{3}\right)$ steps.

\section{FAST RECONSTRUCTION FOR SPARSE FRAMES}

In this section we will see that if the frame is large enough and sparse then we can get fast reconstruction from the absolute values of the frame coefficients. This verifies our intuition that having much more information than necessary should allow for faster reconstruction. The results in this section were communicated to us by M. Strauss [34].

TheOREM 4.1. Let $\mathbb{H}$ be a Hilbert space and let $\left\{e_{1}, \ldots, e_{N}\right\}$ be a fixed orthonormal basis for $\mathbb{H}$.

(a) (Real case) Let $\mathcal{F}$ be the $M=N(N+1) / 2$-element frame

$$
\left\{e_{1}, e_{2}, \ldots, e_{N}\right\} \bigcup\left\{e_{i}+e_{j}\right\}_{1<i<j \leq N}
$$

(b) (Complex case) Let $\mathcal{F}$ be the $M=N^{2}$-element frame

$$
\left\{e_{1}, e_{2}, \ldots, e_{N}\right\} \bigcup\left\{e_{i}+e_{j}, e_{i}+\sqrt{-1} e_{j}\right\}_{1<i<j \leq N}
$$


These frames have unique reconstruction of vectors (up to multiplication by a root of unity) from the absolute values of their frame coefficients with $\leq N$ calculations.

Proof. Let $\mathcal{F}=\left\{f_{1}, \ldots, f_{M}\right\}$ be one of the frames in the statement of the Theorem. Let $x \in \mathbb{H}$ be a vector and let $a_{i}=\left\langle x, f_{i}\right\rangle$ for $i=1,2, \ldots, M$ be the frame coefficients. Since $\left\{e_{1}, \ldots, e_{N}\right\}$ span $\mathbb{H}, a_{i} \neq 0$ for some index $1 \leq i \leq N$. Let $a_{i}$ be the first non-zero frame coefficient and let $b_{j}=a_{j} / a_{i}$ for $i=1,2, \ldots, M$ (so $b_{i}=1$ ). Given $\left\{\left|a_{j}\right|\right\}_{j=1}^{M}$ we will compute the $b_{k}$ 's for $i<k \leq N$ in $N-k$ steps. From this we can recover $x /\left|a_{i}\right|$ and thus the vector $x$ up to a global multiplication by a root of unity.

In the real case we can compute $b_{k}$ from $\left|b_{k}\right|$ and $\left|b_{i}+b_{k}\right|=\left|1+b_{k}\right|$. The formula is

$$
b_{k}=\frac{\left|1+b_{k}\right|^{2}-\left|b_{k}\right|^{2}-1}{2} .
$$

In the complex case we can determine $b_{k}$ from $\left|b_{k}\right|,\left|1+b_{k}\right|$ and $\left|1+\sqrt{-1} b_{k}\right|$. Here the formula is

$$
b_{k}=\frac{1}{2}\left(\left|1+b_{k}\right|^{2}-\sqrt{-1}\left(\left|1+\sqrt{-1} b_{k}\right|^{2}\right)+(\sqrt{-1}-1)\left(1+\left|b_{k}\right|^{2}\right)\right) \text {. }
$$

REMARK 4.2. If choose an orthonormal basis $\left\{e_{1}, \ldots, e_{N}\right\}$ for $\mathbb{H}$ then we can reconstruct the open set of vectors $x$ with $\left\langle x, e_{i}\right\rangle \neq 0$ using the algorithm above and the frame $\left\{e_{1}, \ldots, e_{N}\right\} \bigcup\left\{e_{j}+e_{i}\right\}_{j \neq i}$ if $\mathbb{H}$ is real or $\left\{e_{1}, \ldots, e_{N}\right\} \bigcup\left\{e_{j}+e_{i}, \sqrt{-1} e_{j}+e_{i}\right\}_{j \neq i}$ if $\mathbb{H}$ is complex.

REMARK 4.3. One way to decrease the size of our frames is to use early information to reduce the number of frame vectors used. For example, in the real example above, we can reduce the numbers of vectors used to $2 N-1$ and still do reconstruction with $N$-steps. What we do is compute the absolute value of the inner products $\left\{\left|\left\langle f, e_{i}\right\rangle\right|\right\}_{i=1}^{N}$. Then go to the first non-zero coefficient, say n. Now we just have to compute the frame coefficients against $\left\{e_{n}+e_{m}\right\}_{m=n+1}^{N}$ and we will get quick reconstruction as above with just these coefficients.

\section{ALGORITHMS USING PROJECTIVE 2-DESIGNS}

Next, we examine frames that are not sparse, but provide us with a simple reconstruction formula using only the absolute value of the frame coefficients. This is equivalent to the construction (from the magnitudes of the frame coefficients) of the self-adjoint rank-one operators $Q_{x}, x \in \mathbb{H}$ given by $Q_{x}(y)=\langle y, x\rangle x$, for $y \in \mathbb{H}$. So in this section we will work with the projective space of $\mathbb{H}$.

Definition 5.1. Let $\mathbb{H}$ be a finite-dimensional real or complex Hilbert space and denote by PHI the projective space of $\mathbb{H}$ formed by all orthogonal rank-one projections $\left\{P: P=P^{*} P\right\}$ on $\mathbb{H}$. Equivalently, we can identify each projection by its range in $\mathbb{H}$ and think of this projective space as the set of all one-dimensional subspaces of $\mathbb{H}$.

The projective space $P \mathbb{H}$ is naturally embedded in the space $\mathbb{X}$ containing all self-adjoint rank-one operators. $W e$ define the space of homogeneous $\boldsymbol{k}^{\text {th }}$ degree polynomials $\operatorname{Hom}(k)$ on $P \mathbb{H}$ to consist of functions on $\mathbb{X}$ that can be expressed in the form

$$
h: X \mapsto \operatorname{tr}\left[X^{\otimes k} H\right],
$$

with $H$ an operator on $\mathbb{H}^{\otimes k}$.

We denote by $\mu$ the probability measure on $P \mathbb{H}$ which is invariant under the conjugation of the projections with orthogonal matrices or unitaries in the real or complex case, respectively.

A finite set $\mathbb{X}$ in $P \mathbb{H}$ is called a projective $\boldsymbol{t}$-design [25], $t \in\{1,2, \ldots\}$, if for every homogeneous polynomial $h \in \operatorname{Hom}(k)$ of degree $0 \leq k \leq t$, we have

$$
\int_{P \mathbb{H}} h d \mu=\frac{1}{|\mathbb{X}|} \sum_{P \in \mathbb{X}} h(P),
$$

where $|\mathbb{X}|$ denotes the size of the set $\mathbb{X}$. 
Two known types of examples for projective 2-designs are a maximal equiangular set of lines generated by 2-uniform tight frames and the set of lines generated by a maximal set of mutually unbiased bases in $\mathbb{H}[2]$.

The following theorem from [2] gives a reconstruction formula for $Q_{x}$ that only depends on the magnitudes of the frame coefficients when the frame $\mathcal{F}$ is associated with a projective 2-design. The proof of this theorem will appear elsewhere.

TheOREM 5.2. Let $\mathbb{H}$ be a $N$-dimensional real or complex Hilbert space and $F=\left\{f_{1}, f_{2}, \ldots f_{M}\right\}$ a uniform $M / N$-tight frame such that the orthogonal projections onto the one-dimensional subspaces containing the frame vectors form a projective 2-design in PHH. Given a vector $x \in \mathbb{H}$ with associated self-adjoint rank-one operator $Q_{x}$, then

$$
Q_{x}=\frac{N(N+1)}{M} \sum_{j=1}^{M}\left|\left\langle x, f_{j}\right\rangle\right|^{2} Q_{f_{j}}-\|x\|^{2} I .
$$

REMARK 5.3. The above theorem gives an algorithm that allows us to reconstruct $x$, up to a an overall unimodular constant, by considering one non-vanishing row of the matrix $Q_{x}$. Thus, the computation requires only $O(M N)$ operations. For our earlier examples of maximal 2-uniform frames and mutually unbiased bases that yield projective 2-designs, $M$ is of order $N^{2}$. This results in $O\left(N^{3}\right)$ operations required for reconstruction.

\section{ALGORITHMS USING MUTUALLY UNBIASED BASES}

In this section we will see that for a frame which contains a maximal number of mutually unbiased bases, the reconstruction formula for signal reconstruction without phase is especially simple. The results in this section come from [2] and the proofs will appear elsewhere.

Definition 6.1. Let $\mathbb{H}$ be a complex Hilbert space of dimension $N$. Let $\mathbb{J}=\{1,2, \ldots N+1\}$ and $\mathbb{K}=\{1,2, \ldots N\}$. If the family of vectors $\left\{e_{k}^{j}: j \in \mathbb{J}, k \in \mathbb{K}\right\}$ forms $N+1$ mutually unbiased bases in $\mathbb{H}$ and $\omega$ is a primitive $N$-th root of unity, then we denote

$$
B_{k}^{j}=\frac{1}{\sqrt{N}} \sum_{l=1}^{N} \omega^{k l} P_{l}^{j},
$$

where for each $k \in \mathbb{K}, j \in \mathbb{J}, P_{k}^{j}$ is the rank-one orthogonal projection onto the span of $e_{k}^{j}$.

Now we see how to do signal reconstruction using mutually unbiased bases [2]. The proof will appear elsewhere.

TheOREM 6.2. Given a family of vectors $\left\{e_{k}^{j}, j \in \mathbb{J}, k \in \mathbb{K}\right\}$ that form $N+1$ mutually unbiased bases in $\mathbb{C}^{N}$, a primitive $N$-th root of unity $\omega$ and the associated operators $\left\{B_{k}^{j}\right\}$, then for all $x \in \mathbb{C}^{N}$,

$$
Q_{x}=\frac{\|x\|^{2}}{N} I+\frac{1}{\sqrt{N}} \sum_{j=1}^{N+1} \sum_{k, l=1}^{N} \omega^{-k l}\left|\left\langle x, e_{l}^{j}\right\rangle\right|^{2} B_{k}^{j} .
$$

If we use the discrete chirps then the reconstruction formula simplifies to [2].

Corollary 6.3. If $N$ is prime and we use the discrete chirps constructed earlier as a maximal set of mutually unbiased bases, then the reconstruction formula becomes

$$
x_{n} \overline{x_{1}}=\frac{1}{N}\|x\|^{2} \delta_{n, 1}+\frac{1}{N} \sum_{j=1}^{N+1} \sum_{l=1}^{N} \omega^{-(j-1)\left(n^{2}-1\right)+l(n-1)}\left|\left\langle x, e_{l}^{j}\right\rangle\right|^{2} .
$$




\section{LINEAR TIME RECONSTRUCTION}

As we saw, the use of 2-designs yields algorithms which do reconstruction without phase using $O\left(N^{3}\right)$ operations for an N-dimensional Hilbert space. Using the Fast Fourier Transform gives some improvement for the special case of chirps. In [2] there also appear much faster algorithms requiring on the order of $N$ operations. The construction of these frames involves combining the earlier constructions with the notion of fusion frames [12-14] (see also [7]). The algorithm at first looks like it also needs a number of operations which is quadratic in $N$. But it is then modified so that only a portion of the coefficients are actually used in reconstruction reducing overall the number of calculations to linear in $N$.

\section{CONCLUSION}

We have seen that a generic frame of sufficient size will give signal reconstruction without phase in a polynomial number of steps. We have also seen that for certain tight frames, there are remarkably simple reconstruction algorithms that only use the magnitudes of the frame coefficients. One reason for obtaining a simple expression is the use of projective 2-designs. Since examples for such designs have a small set of possible magnitudes for inner products between frame vectors, the general reconstruction formula can be simplified even further.

Furthermore, we have demonstrated that there are frames for which the number of operations required for reconstruction only grows linearly with the dimension of the Hilbert space.

Reconstruction without phase is also of interest when coefficients are lost, e.g. in the course of a data transmission $[8,15,20,26]$. Since 2-uniform tight frames are in certain situations optimal for a small number of lost coefficients, [8], it may be worthwhile pursuing how well reconstruction without phase performs in the presence of lost coefficients. This topic will be pursued in another work.

\section{ACKNOWLEDGMENTS}

B.G.B. has been partially supported by the Natural Sciences and Research Engineering Council of Canada. P.G.C. acknowledges support from NSF DMS 0704216, D.E. acknowledges support from the University of Missouri Research Board.

\section{REFERENCES}

1. R. Balan. Equivalence relations and distances between Hilbert frames. Proc. Amer. Math. Soc., 127(8):23532366, 1999.

2. R. Balan, B. G. Bodman, P. G. Casazza and D. Edidin, Painless reconstruction from magnitudes of frame coefficients, preprint.

3. R. Balan, P. Casazza, D. Edidin, On signal reconstruction without phase, Appl.Comput.Harmon.Anal. 20 (2006), 345-356.

4. R. Balan, P. Casazza, D. Edidin, Equivalence of Reconstruction from the Absolute Value of the Frame Coefficients to a Sparse Representation Problem, to appear in IEEE Signal Proc. Letters (2007).

5. R. H. Bates and D. Mnyama. The status of practical Fourier phase retrieval, in W. H. Hawkes, ed., Advances in Electronics and Electron Physics, 67:1-64, 1986.

6. C. Becchetti and L. P. Ricotti. Speech recognition theory and $C++$ implementation. Wiley (1999).

7. B. G. Bodmann, Optimal linear transmission by loss-insensitive packet encoding, Appl. Comput. Harmon. Anal. 22 (2007), 274-285.

8. B. G. Bodmann and V. I. Paulsen, Frames, graphs and erasures, Linear Algebra Appl. 404 (2005), 118-146.

9. A. R. Calderbank, P. J. Cameron, W. M. Kantor, and J. J. Seidel, $Z_{4}$-Kerdock codes, orthogonal spreads, and extremal Euclidean line-sets, Proc. London Math. Soc. (3) 75 (1997), no. 2, 436-480.

10. P. J. Cameron and J. J. Seidel, Quadratic forms over GF(2), Indag. Math. 35 (1973), 1-8.

11. P. G. Casazza and M. Fickus, Fourier transforms of finite chirps, EURASIP J. Appl. Signal Process. (2006), Frames and overcomplete representations in signal processing, communications, and information theory, Art. ID $70204,1-7$. 
12. P. G. Casazza and G. Kutyniok, Frames of subspaces, Wavelets, frames and operator theory, Contemp. Math., vol. 345, Amer. Math. Soc., Providence, RI, 2004, pp. 87-113.

13. P. G. Casazza and G. Kutyniok, Robustness of fusion frames under erasures of subspaces and of local frame vectors, Contemporary Math (to appear).

14. P. G. Casazza, G. Kutyniok and S. Li, Fusion frames and distributed processing, preprint.

15. P. Casazza and J. Kovačević, Equal-norm tight frames with erasures, Adv. Comp. Math. 18 (2003), 387-430.

16. O. Christensen, An Introduction to Frames and Riesz Bases, Birkhäuser, Boston, 2003.

17. P. Delsarte, J. M. Goethals, and J. J. Seidel, Spherical codes and designs, Geometriae Dedicata 6 (1977), no. $3,363-388$.

18. J. R. Fienup. Reconstruction of an object from the modulus of its fourier transform, Optics Letters, 3 (1978), $27-29$.

19. J. R. Fienup. Phase retrieval algorithms: A comparison, Applied Optics, 21 (15) (1982), 2758-2768.

20. V. K. Goyal, J. Kovačević, and J. A. Kelner, Quantized frame expansions with erasures, Appl. Comp. Harm. Anal. 10 (2001), 203-233.

21. D. Han and D. Larson. Frames, bases and group representations, Memoirs American Math. Soc. 147 (2000), no. 697.

22. M. H. Hayes. The reconstruction of a multidimensional sequence from the phase or magnitude of its fourier transform. IEEE Trans. ASSP, 30 (2) (1982), 140-154.

23. R. Holmes and V. I. Paulsen, Optimal frames for erasures, Lin. Alg. Appl. 377 (2004), 31-51.

24. S. D. Howard, A. R. Calderbank, and W. Moran, The finite Heisenberg-Weyl groups in radar and communications, EURASIP J. Appl. Signal Process. (2006), no. Frames and overcomplete representations in signal processing, communications, and information theory, Art. ID 85685, 1-12.

25. A. Klappenecker and M. Rötteler, Mutually unbiased bases are complex projective 2-designs, Proc. Int. Symp. on Inf. Theory, IEEE, 2005, pp. 1740-1744.

26. J. Kovačević, P. L. Dragotti, and V. K. Goyal, Filter bank frame expansions with erasures, IEEE Trans. Inform. Theory 48 (2002), 1439-1450.

27. G. Liu. Fourier phase retrieval algorithm with noise constraints, Signal Processing, 21(4):339-347, 1990.

28. A. Peres, Quantum Theory: Concepts and Methods, Kluwer, Dordrecht, 1993.

29. J.G. Proakis. Algorithms for statistical signal processing. Prentice Hall (2002).

30. J. G. Proakis, J. R. Deller and J. H. L. Hansen. Discrete-Time processing of speech signals. IEEE Press (2000).

31. J. M. Renes, R. Blume-Kohout, A. J. Scott, and C. M. Caves, Symmetric Informationally Complete Quantum Measurements, J. Math. Phys., vol. 45, pp. 2171-2180, 2004.

32. L. Rabiner and B. H. Juang. Fundamentals of speech recognition. Prentice Hall Signal Processing Series (1993).

33. R. F. Streater and A. S. Wightman. PCT, Spin and Statistics and All That. Princeton University Press, Landmarks in Mathematics and Physics, 2000.

34. M. Strauss, Private communication.

35. S. V. Vaseghi. Advanced digital signal processing and noise reduction. Wiley (2002).

36. F. W. Warner. Foundations of differential manifolds and Lie groups Graduate Texts in Mathematics 94. Springer-Verlag 1983. 